\title{
On the Scaling Violations of Diffractive Structure Functions: Operator Approach
}

\section{Johannes Blümlein*}

Deutsches Elektronen Synchrotron, DESY Platanenallee 6, D-15738 Zeuthen, Germany E-mail: 'Johannes. Bluemlein@dey.de'

\section{Dieter Robaschik}

Brandenburgische Technische Universität Cottbus, Fakultät 1, PF 101344, D-03013

Cottbus, Germany

E-mail: 'drobasch@physik.tu-cottbus.dei

ABSTRACT: A quantum field theoretic treatment of inclusive deep-inelastic diffractive scattering is given. The process can be described in the general framework of nonforward scattering processes using the light-cone expansion in the generalized Bjorken region. Evolution equations of the diffractive hadronic matrix elements are derived at the level of the twist-2 contributions. The diffractive parton densities are obtained as projections of two-variable parton distributions. An analogous formalism applies to the higher twist contributions.

\section{Introduction}

Deep inelastic diffractive lepton-nucleon scattering was observed at the electron-proton collider HERA some years ago [i]1]. This process is measured in detail by now [2] the structure function $F_{2}^{D}\left(x, Q^{2}\right)$ was extracted. The remarkable experimental observation was (see e.g. [3i] figure 3) that $i$ ) the scaling violations of $F_{2}^{\mathrm{DIS}}\left(x, Q^{2}\right)$ and $F_{2}^{\text {diffr }}\left(x, Q^{2}\right)$ are about the same, and $i$ ) their ratio is of $O(1 / 8 \ldots 1 / 10)$. The reason for the first observation should be perturbative and therefore be explained within perturbative QCD. To obtain also the theoretical understanding for the second aspect, one should find a formulation of the process such that lattice methods can be applied and find out later what the reason for this behavior actually is. Moreover, one should try to find a formulation in which also the higher twists find a field theoretically well-defined place. This is the task of this investigation ${ }^{1}$ and leads us to the operator approach to diffractive scattering.

${ }^{*}$ Speaker.

${ }^{1}$ For details see Ref. ${ }_{4}^{i} \mid$. 


\section{Lorentz Structure}

The differential diffractive scattering cross section

$$
d^{5} \sigma_{\text {diffr }}=\frac{1}{2\left(s-M^{2}\right)} \frac{1}{4} d P S^{(3)} \sum_{\text {spins }} \frac{e^{4}}{Q^{2}} L_{\mu \nu} W^{\mu \nu} .
$$

is characterized by a large rapidity gap $\Delta \eta_{R} \sim 1 / x_{\mathbb{P}}$ between the diffractive jets and the outgoing proton and depends on the variables $x=Q^{2} /\left(W^{2}+Q^{2}-M^{2}\right), t=\left(p_{1}-p_{2}\right)^{2}, M_{X}^{2}=$ $\left(q+p_{1}-p_{2}\right)^{2}$ and the virtuality of the momentum transfer $Q^{2}$. Here $x$ is the Bjorken variable, $W^{2}=\left(q+p_{1}\right)^{2}$ the hadronic mass squared, $M_{X}$ the diffractive mass, $p_{1,2}$ are the in- and out-going proton momenta. The hadronic tensor for unpolarized scattering depends on four structure functions

$$
\begin{aligned}
W_{\mu \nu}= & \left(-g_{\mu \nu}+\frac{q_{\mu} q_{\nu}}{q^{2}}\right) W_{1}+\left(p_{1 \mu}-q_{\mu} \frac{p_{1} \cdot q}{q^{2}}\right)\left(p_{1 \nu}-q_{\nu} \frac{p_{1} \cdot q}{q^{2}}\right) \frac{W_{3}}{M^{2}} \\
& +\left(p_{2 \mu}-q_{\mu} \frac{p_{2} \cdot q}{q^{2}}\right)\left(p_{2 \nu}-q_{\nu} \frac{p_{2} \cdot q}{q^{2}}\right) \frac{W_{4}}{M^{2}} \\
+ & {\left[\left(p_{1 \mu}-q_{\mu} \frac{p_{1} \cdot q}{q^{2}}\right)\left(p_{2 \nu}-q_{\nu} \frac{p_{2} \cdot q}{q^{2}}\right)+\left(p_{2 \mu}-q_{\mu} \frac{p_{2} \cdot q}{q^{2}}\right)\left(p_{1 \nu}-q_{\nu} \frac{p_{1} \cdot q}{q^{2}}\right)\right] \frac{W_{5}}{M^{2}}, }
\end{aligned}
$$

Since we are going to view the diffractive process in the formalism for non-forward scat-

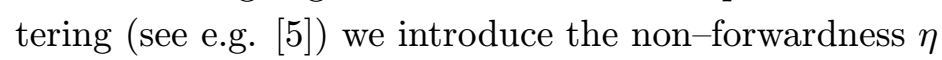

$$
\eta=\frac{q \cdot\left(p_{2}-p_{1}\right)}{q \cdot\left(p_{2}+p_{1}\right)}=-\frac{x_{\mathbb{P}}}{2-x_{\mathbb{P}}} \epsilon\left[-1, \frac{-x}{2-x}\right]
$$

which is directly related to the variable $x_{\mathbb{P}}$.

Most of the data are situated at low values of $t$. Therefore we study the case $t, M^{2} \sim 0$, in which $p_{2}$ and $p_{1}$ are related by $p_{2}=\left(1-x_{\mathbb{P}}\right) p_{1}$. Out of the above four structure functions only two remain. To be able to apply quantum field theoretic methods to the process of diffractive scattering we consider the generalized Bjorken limit

$$
p_{1} . q, \quad p_{2} . q, \quad Q^{2} \rightarrow \infty, \quad x, x_{\mathbb{P}} \text { fixed. }
$$

In this kinematic domain the light-cone dominates and we can consider the process in the light-cone expansion. Together with the use of Mueller's optical theorem it is this property which assures factorization at the level of twist-2 as also found in [i]"], but allows, more than this, the extension of the formalism to higher twist.

\section{The Compton Amplitude}

In inclusive diffractive scattering two color-neutral pieces of the hadronic final state are very well separated in rapidity: the diffractive proton and the diffractive jet-system. On the Lorentz-level one therefore may rewrite the process using Mueller's generalized optical theorem [i[;] which is depicted as follows : 

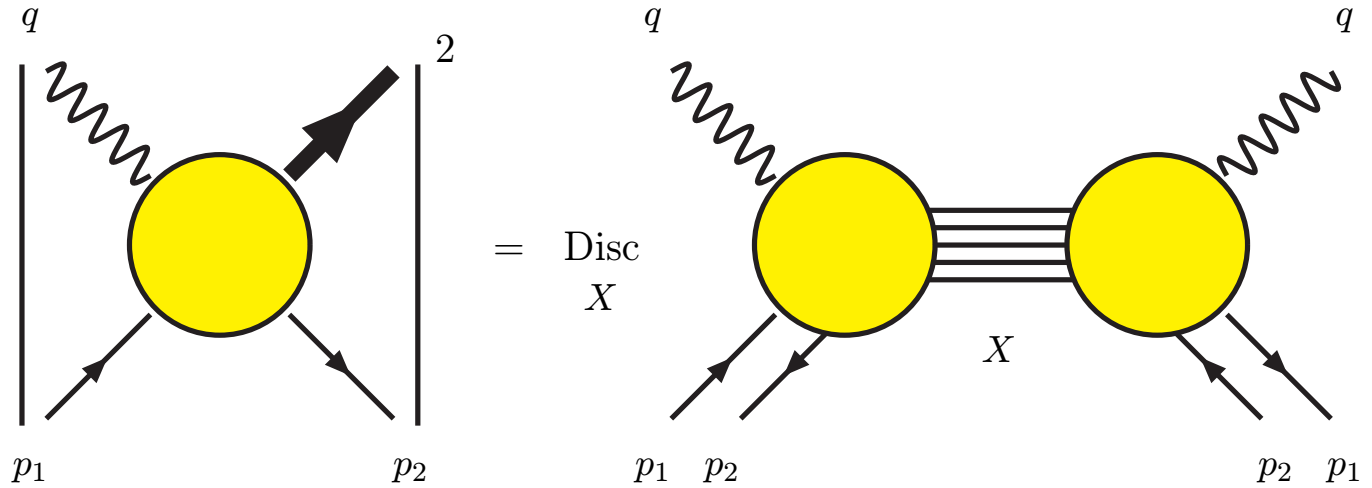

Figure 1: A. Mueller's optical theorem.

The final-state proton is rotated into an initial state antiproton and diffractive scattering can thus be viewed as deep-inelastic scattering off the state $\left\langle p_{1},-p_{2}\right|$. The associated parton densities are derived from the two-variable distribution functions which emerge in the non-forward formalism and are related to the expectation value of the scalar quark operator, cf. [i]

$$
\left\langle p_{1},-p_{2}\left|O^{q}\left(\kappa_{+} x, \kappa_{-} x\right)\right| p_{1},-p_{2}\right\rangle=x p_{-} \int D z \mathrm{e}^{-i \kappa \kappa_{-} x p_{z}} f^{q}\left(z_{+}, z_{-}\right),
$$

with $p_{ \pm}=p_{2} \pm p_{1}$ and $p_{z}=p_{+} z_{+}+p_{-} z_{-}$. Here we retain only the dominant contribution.

The calculation of the twist -2 contribution in lowest order implies the diffractive variant of the Callan-Gross relation, which differs from that in the deep-inelastic case

$$
\mathrm{F}_{2}\left(\beta, \eta, Q^{2}\right)=2 x \mathrm{~F}_{1}\left(\beta, \eta, Q^{2}\right) \equiv F_{2}^{D(3)}\left(x, Q^{2}, x_{\mathbb{P}}\right),
$$

where $\beta=x / x_{\mathbb{P}}$. Finally the diffractive parton densities are related to the two-particle

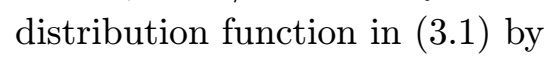

$$
\begin{aligned}
& f^{D}\left(\beta, Q^{2}, x_{\mathbb{P}}\right)=-\int_{-\frac{x_{\mathbb{P}}+2 x}{2-x_{\mathbb{P}}}}^{-\frac{x_{\mathbb{P}}-2 x}{x_{\mathbb{P}}}} d \rho f\left(\rho, 2 \beta+\rho\left(2-x_{\mathbb{P}}\right) / x_{\mathbb{P}}, Q^{2}\right) \\
& \bar{f}^{D}\left(\beta, Q^{2}, x_{\mathbb{P}}\right)=-\int_{-\frac{x_{\mathbb{P}}+2 x}{2-x_{\mathbb{P}}}}^{-\frac{x_{\mathbb{P}}-2 x}{2-x_{\mathbb{P}}}} d \rho f\left(\rho,-2 \beta+\rho\left(2-x_{\mathbb{P}}\right) / x_{\mathbb{P}}, Q^{2}\right) .
\end{aligned}
$$

\section{Evolution Equations}

To derive the twist -2 evolution equation of the diffractive structure functions we start from the general evolution equation for operators on the light cone, see $[\bar{F}]$. The all-order singlet anomalous dimension matrix is $\gamma^{\mathrm{AB}}\left(\kappa_{+}, \kappa_{-}, \kappa_{+}^{\prime}, \kappa_{-}^{\prime} ; \mu^{2}\right)$, where $\kappa_{ \pm}$are light-cone marks and $\mu^{2}$ denotes the factorization scale. Forming the evolution equations being associated to the matrix element $\left\langle p_{1},-p_{2}\left|O^{q}\right| p_{1},-p_{2}\right\rangle$ one notices that the anomalous dimensions are independent of $\kappa_{+}$which can be set to 0 . Furthermore, the all-order rescaling relation

$$
\gamma^{A B}\left(\kappa_{+}, \kappa_{-}, \kappa_{+}^{\prime}, \kappa_{-}^{\prime} ; \mu^{2}\right)=\sigma^{d_{A B}} \gamma^{A B}\left(\sigma \kappa_{+}, \sigma \kappa_{-}, \sigma \kappa_{+}^{\prime}, \sigma \kappa_{-}^{\prime}\right)
$$


applies, where $d_{A B}=2+d_{A}-d_{B}$ and $d_{q}=1, d_{G}=2$. As shown in [i [i] $]$ an evolution equation may be obtained for the diffractive parton densities $f^{A}\left(\vartheta, \eta, \mu^{2}\right)$, where $\vartheta=z_{-}+z_{+} / \eta$ is the characteristic internal momentum fraction. These distributions have a much wider support than $[0,1]$ in this variable. Upon taking the absorptive part, $\vartheta=2 \beta$ holds and the final twist-2 evolution equations read

$$
\mu^{2} \frac{d}{d \mu^{2}} f_{A}^{D}\left(\beta, x_{\mathbb{P}} ; \mu^{2}\right)=\int_{\beta}^{1} \frac{d \beta^{\prime}}{\beta^{\prime}} P_{A}^{B}\left(\frac{\beta}{\beta^{\prime}} ; \mu^{2}\right) f_{B}^{D}\left(\beta^{\prime}, x_{\mathbb{P}} ; \mu^{2}\right) .
$$

\section{Conclusions}

The scattering cross section for unpolarized diffractive $e p$ scattering depends on four structure functions. For $t \sim 0$ only two structure functions emerge. In the operator approach the process can be interpreted as deeply inelastic scattering off a state $\left\langle p_{1},-p_{2}\right|$ formed out of an initial state proton of momentum $p_{1}$ and an antiproton of momentum $p_{2}$ using Mueller's optical theorem. The Callan-Gross relation gets modified. The evolution of the twist -2 contributions to the diffractive structure functions was shown to be forward and to affect only $\beta$, which compares to Bjorken $x$ in the deep-inelastic case. The second dimensionless variable describing the process, $x_{\mathbb{P}}$ resp. the non-forwardness $\eta$, behaves as a pure parameter and does not contribute to the evolution kernels. The present method applies analogously to the contributions of higher twist, as long as light-cone dominance can be assured. It is desirable to measure the operator matrix elements being derived using lattice methods and to compare them to those of the deep-inelastic case. In this way the value of the respective ratio being measured by experiment might be understood in the future.

\section{Acknowledgments}

We would like to thank our experimental colleagues, in particular John Dainton, for their interest in the present work and look forward to the forthcoming analyzes of the high statistics HERA data to test this approach.

\section{References}

[1] M. Derrick et al., ZEUS collaboration, Phys. Lett. B315 (1993) 481;

T. Ahmed et. al., H1 collaboration, Nucl. Phys. B429 (1994) 477.

[2] J. Breitweg et al., ZEUS collabortaion, Eur. Phys. J. C6 (1999) 43;

C. Adloff et al., H1 collaboration, Z. Phys. C76 (1997) 613.

[3] H. Abramowicz and J. Dainton, J. Phys. G22 (1996) 911.

[4] J. Blümlein and D. Robaschik, Phys. Lett. B517 (2001) 222.

[5] J. Blümlein, B. Geyer, and D. Robaschik, Nucl. Phys. B560 (1999) 283.

[6] A. Berera and D.E. Soper, Phys. Rev. D50 (1994) 4328; D53 (1996) 6162;

J. Collins, Phys. Rev. D57 (1998) 3051; Erratum: D61 (2000) 019902;

F. Hautmann, Z. Kunszt, and D.E. Soper, Nucl. Phys. B563 (1999) 153.

[7] A.H. Mueller, Phys. Rev. D2 (1970) 2963; Phys. Rev. D4 (1971) 150;

P.D.P. Collins, An Introduction to Regge Theory and High Energy Physics, (Cambridge University Press, Cambridge, 1977), pp. 331. 